\title{
Multi-kilowatt Single-mode Ytterbium-doped Large-core Fiber Laser
}

\author{
Yoonchan Jeong*, Alexander J. Boyland, Jayanta K. Sahu, Seunghwan Chung, \\ Johan Nilsson, and David N. Payne \\ Optoelectronics Research Centre, University of Southampton, Southampton SO17 1BJ, \\ United Kingdom
}

(Received November 9, 2009 : revised November 23, 2009 : accepted November 23, 2009)

We have demonstrated a highly efficient cladding-pumped ytterbium-doped fiber laser, generating $>2.1 \mathrm{~kW}$ of continuous-wave output power at $1.1 \mu \mathrm{m}$ with $74 \%$ slope efficiency with respect to launched pump power. The beam quality factor $\left(\mathrm{M}^{2}\right)$ was better than 1.2. The maximum output power was only limited by available pump power, showing no evidence of roll-over even at the highest output power. We present data on how the beam quality depends on the fiber parameter, based on our current and past fiber laser developments. We also discuss the ultimate power-capability of our fiber in terms of thermal management, Raman nonlinear scattering, and material damage, and estimate it to $10 \mathrm{~kW}$.

Keywords : High-power fiber laser, Ytterbium-doped fiber, Optical power scaling, Single-mode fiber laser

OCIS codes : (140.3510) Lasers, fiber; (140.3480) Lasers, diode-pumped

\section{INTRODUCTION}

We have seen dramatic advances of high-power fiber laser and amplifier technology in the past few years [1-3]. In particular, the research on ytterbium $\left(\mathrm{Yb}^{3+}\right)$-doped fibers (YDFs) has been intense because of their outstanding efficiency, power-handling characteristics, and low cost of ownership for various scientific and industrial applications. These include materials processing, defense, remote sensing, range-finding, free-space communication, display (when converted into visible), lithography (when converted into ultraviolet), bio-medical applications, etc. [4, 5]. Unlike neodymium, erbium, and thulium ions which are also often chosen as a dopant for high-power fiber lasers, $\mathrm{Yb}^{3+}$ has a very simple structure of energy levels without excited-state absorption or cross-relaxation. On top of this, a small quantum defect between the pump (910-980 nm) and signal (1030-1100 nm) wavelengths leads to high efficiency that can exceed $80 \%$, and in turn significantly reduces heat generation. $\mathrm{Yb}^{3+}$ is superior also in this aspect, which, despite the superb thermal properties of the fiber geometry, becomes increasingly important as the power levels continue to increase. Furthermore, high doping concentration is readily achievable without

\footnotetext{
*Corresponding author: yoj@orc.soton.ac.uk
}

concentration quenching. This is a key requirement for cladding-pumping, to keep device lengths reasonably short for suppression of undesired nonlinear scattering, e.g. stimulated Raman scattering (SRS), and stimulated Brillouin scattering (SBS). While photo-darkening can raise some short- and long-term reliability issues [6-8], this can be controlled through the choice of operating regime and host [7]. Thus, YDFs remain most attractive for multi-kW power applications.

YDFs can be power-scaled in laser (resonator) configurations [1, 2] as well as in amplifier configurations [3]. Laser configurations are attractive due to simplicity and compactness. On the other hand, amplifier configurations allow for more refined control, such as in master-oscillator - power amplifiers for single-frequency signals [9], controlled pulses [10], etc. However, they normally require multiple amplification stages to reach multi-kW power levels, thereby resulting in relatively complicated structures. Furthermore isolators and/or wavelength filters are required unless the gain is sufficiently low, and obtaining such components of high-power capability presents an additional difficulty. Thus, for the purpose of power-scaling, we have focused our work on a single-fiber-based laser configuration.

Since the demonstration of the first kilowatt quasisingle-mode $\left(\mathrm{M}^{2}=1.4\right) \mathrm{YDF}$ laser in 2004 [1], only a limited number of publications have reported on the 
further development of fiber lasers beyond a kilowatt of output power: $1.53 \mathrm{~kW}$ output power from a single Yb-doped photonic crystal fiber laser was reported in 2005 while the output beam was slightly multimode [2]. Apart from such YDF-based single-fiber laser results, IPG Photonics has recently announced that $10 \mathrm{~kW}$ of output power in a single-mode beam was achieved from a chain of multiple low-gain YDF amplifiers where the last stage was pumped by fiber lasers (via a tandempumping scheme) [3]. However, its technical details are hardly available in the literature while $1.5 \mathrm{~kW}$ systems are now commercially available from the company [3].

Here we report a single-fiber-based ytterbium-doped fiber laser (YDFL) in a simple end-pumped configuration with near diffraction-limited $\left(\mathrm{M}^{2}=1.2\right)$, multikilowatt output power $(>2.1 \mathrm{~kW})$, based on a state of the art, low-index polymer-clad YDF. We emphasize the simplicity of a directly diode-pumped configuration for achieving multi-kilowatt single-mode output power in comparison with other configurations, e.g., multistage amplifiers or tandem-pumping schemes. We discuss the correlation between the beam quality and the fiber parameter, based on our current and past fiber laser developments. In addition, we analyze the ultimate power limits of this fiber, which indicate that this simplest of fiber laser configurations is well suited to power-scaling, and may well be capable of generating $>10 \mathrm{~kW}$ output power.

\section{EXPERIMENTS AND RESULTS}

We fabricated a preform in-house by the modified chemical-vapor-deposition (MCVD) and solution-doping technique. In order to obtain efficient mode-mixing of the pump beam, we milled two sides of the preform in parallel, symmetrically with respect to the fiber core. As a result, the preform became "double-D-shaped" with a short-axis length of approximately $90 \%$ of the other. We pulled the preform into a fiber: The fiber had an $850 \mu \mathrm{m}$ diameter in the long axis of the silica inner cladding. The fiber was coated with a low-refractiveindex polymer which provided a nominal inner-cladding numerical aperture (NA) of 0.48 . The fiber had a $50-\mu \mathrm{m}$ diameter $\mathrm{Yb}^{3+}$-doped aluminosilicate core, and its nominal NA was below 0.06 . The choice of fiber parameters and fabrication is a crucial part of our research since it determines the beam quality as well as the powerhandling. We made sure that there was no central dip in the refractive-index profile (RIP) of the core. This is essential for achieving good beam quality with such a large core dimension. A central refractive-index dip that often results after the MCVD process, without proper care, leads to a donut-shaped fundamental mode [11]. Such a mode is difficult to handle, and in any case results in an output beam that is not diffraction-limited. Still, even without a central dip, with a Gaussian-like fundamental mode, suppression of higher-order modes is challenging. Selective doping in the radial direction [12] and mode-filtering via bending the low-NA gain fiber [1] can help in obtaining good beam quality, by significantly reducing the net gain for higher-order modes. However, selective, and therefore restricted, doping increases the pump absorption length. This increases the impact of nonlinearities such as stimulated Raman scattering (SRS). Furthermore, bend-induced mode filtering can lead to problematic modal distortions [13]. Therefore, we did not use selective doping, and used a large bend radius in order to avoid significant modal distortions. We estimate that the resultant mode-field areas of the fundamental mode $\left(\mathrm{LP}_{01}\right)$ and the first higher-order mode $\left(\mathrm{LP}_{11}\right)$ are $411 \mu \mathrm{m}^{2}$ and 823 $\mu \mathrm{m}^{2}$, respectively. The small-signal absorption coefficient of the inner cladding was $1 \mathrm{~dB} / \mathrm{m}$ at $976 \mathrm{~nm}$. This corresponds to an $\mathrm{Yb}^{3+}$-concentration of approximately $3700 \mathrm{ppm}$ by weight. This concentration level allows for a low NA and helps in mitigating photo-darkening. The fiber length used in the laser experiments was $20 \mathrm{~m}$.

The experimental setup is shown in Fig. 1. We launched the pump beams through both ends of the

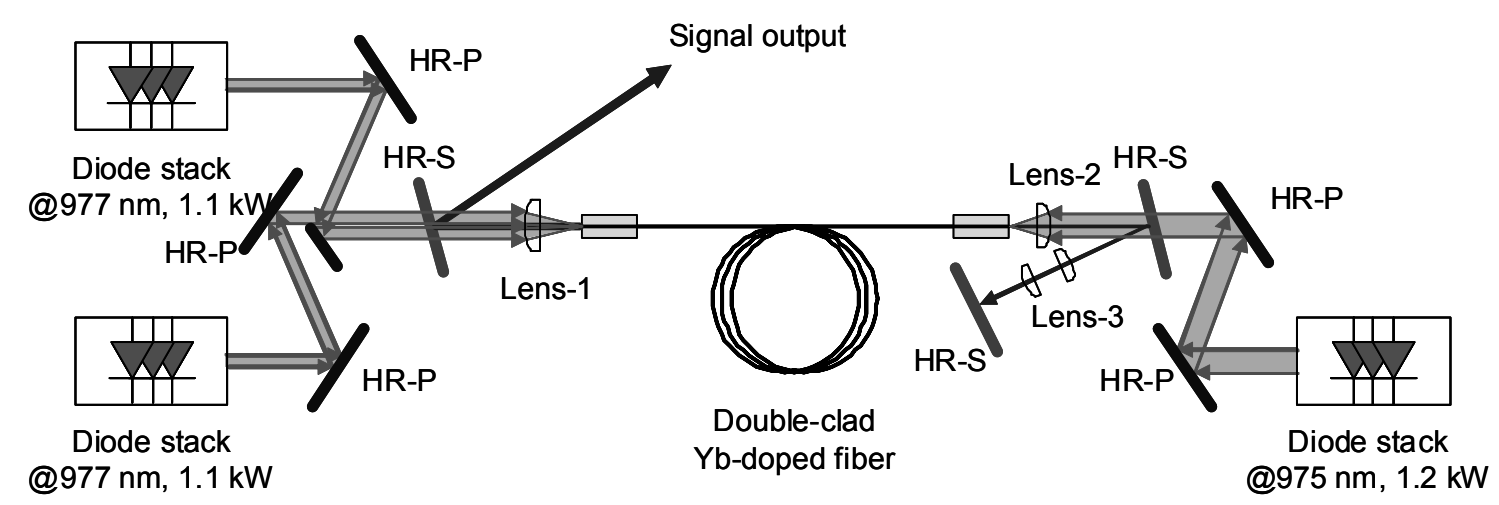

FIG. 1. Experimental arrangement for an YDF laser with diode-stack pump sources. HR-P: high reflectivity at pump wavelength, HR-S: high reflectivity at signal wavelength. 
fiber, via a combination of optics, including lenses and dichroic mirrors. Three diode-laser-stack-based sources were used: Two of them, each emitting $1.1 \mathrm{~kW}$ at 977 $\mathrm{nm}$, were spatially beam-combined into one beam. We obtained coupling efficiencies of over $85 \%$ from the beam-combined end and over $90 \%$ from the other. Both ends of the fiber were polished perpendicular to the fiber axis, without any further processing. At one end of the laser cavity, high-reflectivity feedback was provided by a pair of dichroic mirrors having high transmission at the pump wavelength and high reflection at the signal wavelength. We also used a pair of lenses as a telescope in order to achieve a high degree of collimation of the feedback signal beam. The laser output coupler was formed by the $4 \%$ reflecting flatpolished facet at the other end of the fiber. The signal was separated from the pump beam using another dichroic mirror having the same characteristics as the feedback mirror. Both ends of the fiber were held in temperature-controlled metallic $\mathrm{V}$-grooves designed to prevent possible thermal damage to the fiber coating by any non-guided pump or signal power, or by the heat generated in the laser itself. The fiber was coiled in a cartridge having a $25-\mathrm{cm}$ diameter. Note that except for the careful alignment of the feedback mirror, there was no other mode control other than that inherent to the fiber.

The laser output power characteristics are shown in Fig. 2, together with the output spectrum at full pump power, showing a broad emission linewidth as typical for high-power YDFLs without any wavelength selection. The laser generated a single-ended output in excess of $2.1 \mathrm{~kW}$ at the full pump power. It is noteworthy that the laser output increased linearly with the launched pump power and showed no evidence of roll-over even at the highest output power. The maximum output power was only limited by available pump power. There was no signature of SRS even at the maximum output power. There were no fiber or coating failures due to the thermal load by the absorbed pump. It showed excellent conversion efficiency: The slope efficiency was $74 \%$ with respect to launched pump power. The pump leakage was less than $4 \%$ in total. The resultant quantum efficiency was close to $90 \%$. We measured the short-term temporal characteristics via a fast photodetector of $1 \mathrm{GHz}$ bandwidth and an oscilloscope of $400 \mathrm{MHz}$ bandwidth: The standard deviation of the temporal power fluctuation was below $1.6 \%$. We measured the beam quality factor $\left(\mathrm{M}^{2}\right)$ to better than 1.2 , proving that the output beam was very near single-mode and diffraction-limited. This more than doubles the brightness of our previous result [1]. We attribute this to the enhanced RIP of the fiber core with a sufficiently low NA. The output beam profile is presented in Fig. 3, which shows clean Gaussian intensity patterns for both axes.

Here we further discuss the beam quality characteristics of large-core fiber laser, based on our current and past fiber laser developments $[1,11,14-16]$ : We have found that the beam quality of a fiber laser has a strong correlation with the RIP of the fiber as well as with the $\mathrm{V}$-number. While the V-number itself determines the number of guided modes in the core [20], the number of "lasing" modes is, in fact, determined by the gain that the individual guided mode sees based on the spatial gain profile. That is, the spatial gain profile together with the RIP plays a crucial role. We plot the beam quality factors of our various fiber lasers developed in house as a function of the V-number in Fig. 4, assuming that the fiber cores are simply step-indexed. While we can see that relatively good beam quality, e.g. $\mathrm{M}^{2}<2$ could be obtained if the $\mathrm{V}$-number was less

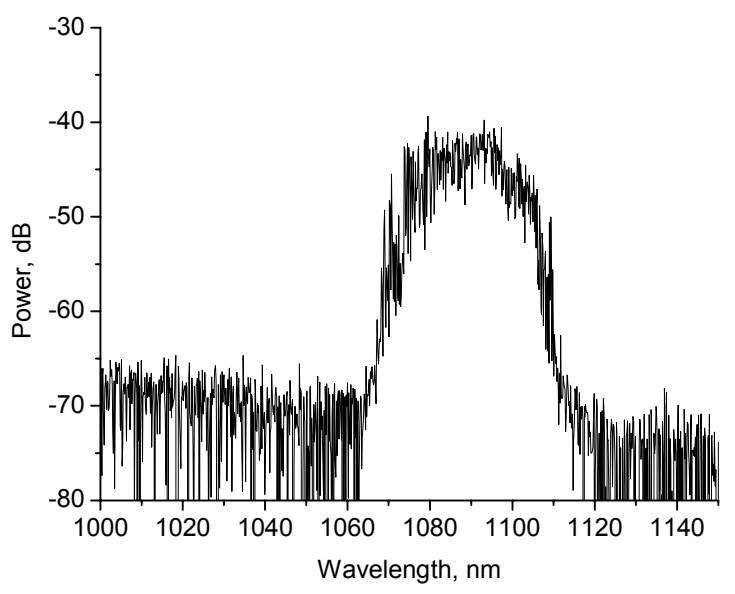

(b)

FIG. 2. (a) Fiber laser output power vs. launched pump power. The slope efficiency is $74 \%$ with respect to launched pump power. (b) Laser output spectrum at full power. Spectral resolution: $1 \mathrm{~nm}$. 


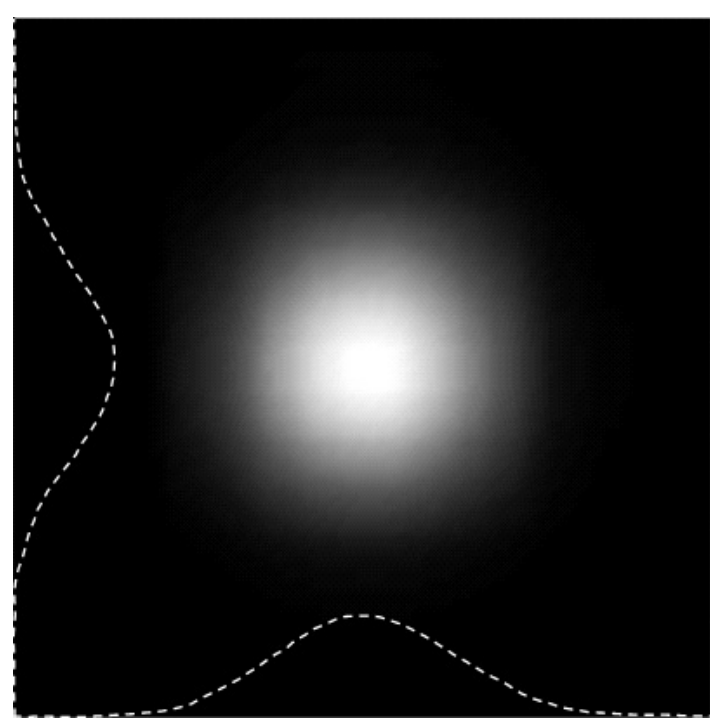

FIG. 3. Output beam profile.

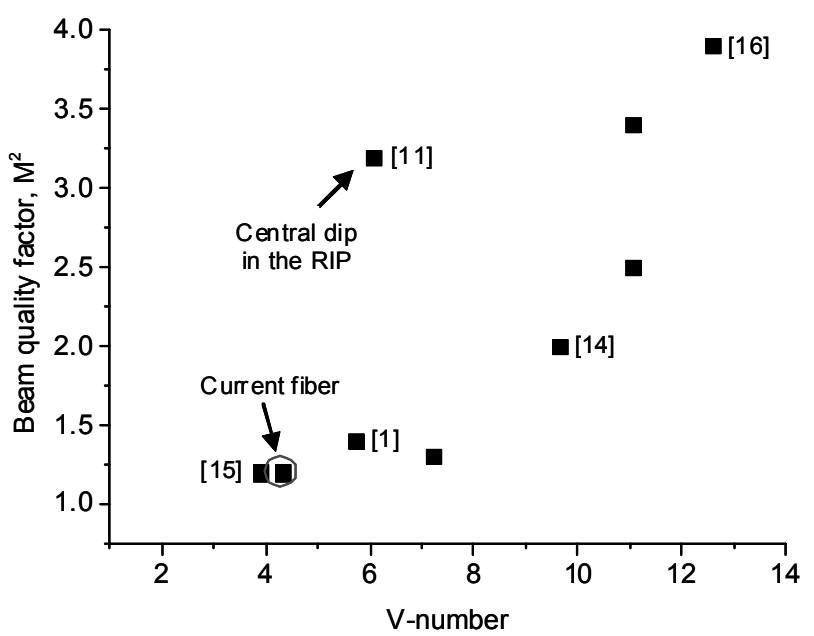

FIG. 4. Beam quality factor $\left(\mathrm{M}^{2}\right)$ vs. V-number: Some data points are labeled according to the reference in which they appeared. References for the non-labeled points can be found in Ref. [1].

than 8, some non-favorable RIP, e.g. an RIP containing a central dip can significantly degrade the beam quality even with quite low a V-number [11]. Since the transverse beam profile of a large-core fiber generally follows the RIP, an unusual shape of RIP, e.g. a central dip or a top-hat with "ears", is deleterious to beam quality. In fact, if the distribution of the doped ions roughly follows the RIP (which is not always true), such RIPs can lead to poor gain-discrimination between the fundamental mode and higher-order modes, so that some higher-order modes can lase together with the fundamental mode. Furthermore, even if only the fundamental mode lases, it results in a non-Gaussianshape output beam that is not diffraction-limited.

We estimate the "effective" V-number of our current fiber to be approximately 4.3, assuming a step-indexed core that has the same effective area for the fundamental mode as that of our fiber. The data point, the beam quality factor of $\mathrm{M}^{2}=1.2$ at $\mathrm{V}=4.3$ from our current fiber, is in line with our previous observations. While it is hard to obtain a V-number of less than 2.405 from a large-core fiber without incurring large bending loss, our results suggest that a large-core fiber with a V-number of about 4 with a proper RIP can effectively operate very near single-mode.

Since our fiber was pump-limited, it is worthwhile to estimate its further power-scalability, particularly given that our diode-stack-based pumps are significantly less bright than present-technology diodes. For example, currently available $200 \mathrm{~W}$ fiber-coupled diode sources with a $200 \mu \mathrm{m}$ core diameter and 0.22 NA can readily be beam-combined into a higher-power single beam by using lenses or possibly by transmission through a tapered fiber bundle [17]. Although we did require a $850 \mu \mathrm{m}$ diameter inner cladding in order to obtain good coupling from our diode sources, the fiber can readily accommodate $>15 \mathrm{~kW}$ of pump power in a dual-end pumping configuration if such state-of-the-art diode pump sources are used, with preserved pump brightness. With this background, we next estimate the further power-scalability of our fiber, with respect to thermal management, Raman nonlinear scattering, and material damage which are the key limiting factors.

Firstly, we consider the thermal characteristics of our fiber at $10 \mathrm{~kW}$ of output power: Fiber lasers, in general, have good thermal characteristics in terms of heat dissipation because of their intrinsic large surfaceto-active-volume ratio; however, multi-kW fiber lasers indeed require careful thermal management [18, 19].

Generally, for fibers, the temperature variation across a fiber section is much smaller than for other geometries thanks to the long and thin fiber geometry. This, combined with basic guided-mode properties, protects against beam distortions due to thermal lensing, which is a major problem for bulk solid-state lasers. Stress fracture, which is another big issue for bulk lasers, is again alleviated by the fiber geometry and the small transverse temperature variations [18]. Most critical for fiber lasers with a low-index polymer outer cladding like that of our fiber, is thermal damage of the outer polymer (maximum durable temperature $<200^{\circ} \mathrm{C}$ ). Because of the lower thermal conductivity of the polymer coating, the inner surface of the coating reaches a temperature which is a significant fraction of that of the silica inner cladding and core, even when the outer surface of the fiber is effectively cooled down. Since the silica inner cladding and core themselves have considerably higher temperature limits [18], the temperature of the inner surface of the polymer coating is, consequently, considered to be critical. The temperature increase across the coating thickness is approximately given by [18] 


$$
\Delta T_{\text {coating }} \approx \frac{Q_{\text {thermal }} \cdot t_{\text {coating }}}{2 \pi r_{\text {silica }} \cdot k_{\text {coating }}},
$$

where $\mathrm{Q}_{\text {thermal }}, \mathrm{t}_{\text {coating, }}, \mathrm{r}_{\text {silica, }}$, and $\mathrm{k}_{\text {coating }}$ represent the thermal power produced by the fiber per unit length, the thickness of the coating, the radius of the silica inner cladding, and the thermal conductivity of the coating, respectively. We assume that the outside of the fiber is kept near room temperature by good heat-sinking arrangements, e.g., water-assisted cooling. For a good margin to the coating damage temperature, in the range between 150 to $200^{\circ} \mathrm{C}$, we assume that the maximum temperature difference across the coating, $\Delta T_{\text {coating }}$ is $70 \mathrm{~K}$. Then, we obtain approximately $\mathrm{Q}_{\text {thermal }} \approx 300 \mathrm{~W} / \mathrm{m}$ after putting the parameters of our fiber into the equation. Figure 5 shows the resulting temperature profile. Based on $1 \mathrm{~dB} / \mathrm{m}$ of pump absorption and $>80 \%$ conversion efficiency with respect to absorbed pump power, the pump power that generates $\mathrm{Q}_{\text {thermal }} \approx 300 \mathrm{~W} / \mathrm{m}$ becomes $6.7 \mathrm{~kW}$ per fiber end. The total pump power can be doubled with dual-end pumping, showing that the output power can readily reach $10 \mathrm{~kW}$ without hitting the thermal limit on output power. Note that the aforementioned, maximum tolerable pump power can readily be coupled into an $850-\mu \mathrm{m}$ diameter inner cladding with an NA of 0.48 if state-of-the-art pump sources are used [17].

A thinner coating is preferable for further improving the thermal management, as it reduces the temperature difference across the coating. We can reduce the coating thickness down to 40 to $50 \mu \mathrm{m}$ without deteriorating the fabrication yield and pump NA. Thus, thermally induced coating damage does not appear to be a major concern for a $10 \mathrm{~kW}$ fiber laser with appropriate heatsinking. This is true even with end-pumping, with the thermal load concentrated to the fiber ends.

Secondly, we consider stimulated Raman scattering. As the pump absorption rate of our fiber is $1 \mathrm{~dB} / \mathrm{m}$ at

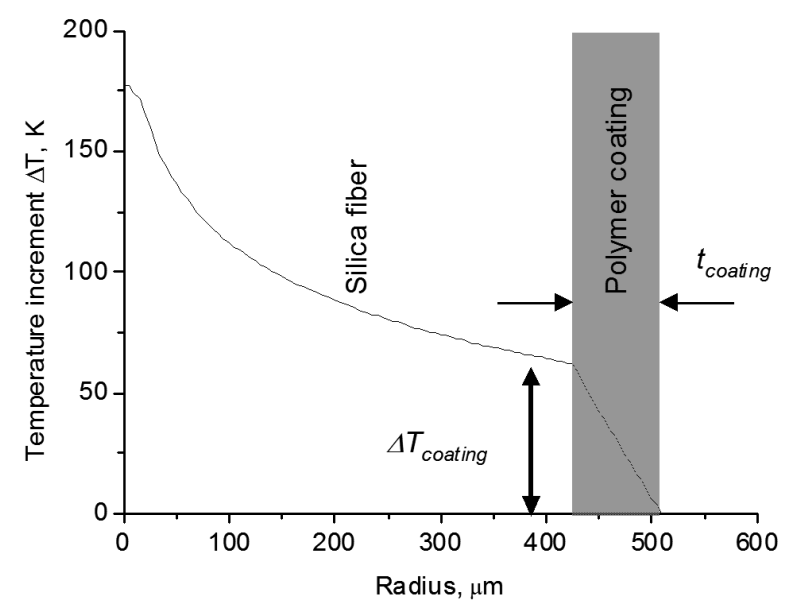

FIG. 5. Estimated temperature profile across the fiber section at $10 \mathrm{~kW}$ output power.
$976 \mathrm{~nm}$, we can set the fiber length at $15 \mathrm{~m}$ to $20 \mathrm{~m}$ in a dual-end pumping configuration. Then, we estimate that the effective length of the laser becomes between $8.6 \mathrm{~m}$ and $11.8 \mathrm{~m}$. Given that the Raman gain coefficient is as large as $1 \times 10^{-13} \mathrm{~m} / \mathrm{W}$ for polarized light [20], the resultant single-pass Raman gain at $10 \mathrm{~kW}$ of output power with random polarization becomes approximately $10 \mathrm{~Np}$ to $14 \mathrm{~Np}$. While these values are still lower than the critical gain of $16 \mathrm{~Np}$ for the forward SRS [20], the margin to spurious feedback at the Raman Stokes wavelength is small. In particular, this becomes more serious in case of the 20-m fiber length. Thus, it is better to set the fiber length at $15 \mathrm{~m}$, and then minimize the feedback at the Raman Stokes wavelength. It is also desirable to center the pump wavelength exactly at $976 \mathrm{~nm}$ to maximize the pump absorption. Then, we predict that stimulated Raman scattering will be controllable even at $10 \mathrm{~kW}$ of output power.

Finally, we consider the core damage: Based on 411 $\mu \mathrm{m}^{2}$ of the estimated effective mode area of our fiber, $10 \mathrm{~kW}$ of output power would lead to the effective core intensity of approximately $24.3 \mathrm{~W} / \mathrm{\mu m}^{2}$. In the literature the damage threshold of silica fibers varies in a wide range of $\sim 20 \mathrm{GW} / \mathrm{cm}^{2}$ to $\sim 600 \mathrm{GW} / \mathrm{cm}^{2}$ [21-22], which indicates that there is huge uncertainty in determining the damage threshold: The damage threshold also depends on whether the signal is a continuous wave $(\mathrm{cw})$ or pulse, and for the pulse it also varies with the pulse width. Also, the damage threshold must be dependent of the condition of the fiber facet, dopant composition, impurity, loss, etc. More recently it has been reported that the damage threshold of ytterbiumdoped silica with a high level of doping concentration (1\% by weight) has a damage threshold similar to that of pure silica which was measured to be $\sim 470 \mathrm{GW} / \mathrm{cm}^{2}$ with 7.5-ns pulses at the illumination wavelength of $1064 \mathrm{~nm}$ [22].

In general, for pulses longer than $\sim 100 \mathrm{ps,} \mathrm{it} \mathrm{is} \mathrm{known}$ that conventional heat deposition causes damage via melting and boiling [23]. Thus, in a cw regime the heat deposition by the pump and signal must be the key factor to determine the core damage. We have estimated that the heat deposition of $\mathrm{Q}_{\text {thermal }} \approx 300 \mathrm{~W} / \mathrm{m}$ at $10 \mathrm{~kW}$ output power keeps the core temperature below $200^{\circ} \mathrm{C}$ which is far below the melting point of silica of over $1900^{\circ} \mathrm{C}$ [18] (see Fig. 5). Therefore, such a thermal load will not destroy the core of our fiber, as long as it is controlled. However, it is noteworthy that our estimation on the fiber temperature is based on the assumption that the outside of the fiber is kept near room temperature by good heat-sinking arrangements, e.g. water-assisted cooling. If it is only cooled down by natural convection $[19,24]$, the core temperature will be much higher and the excessive heat can damage the core, as was observed in Ref. [25]. Therefore, the material damage is also 
strongly correlated with the heat management, and thus, a good heat-sinking arrangement is also very important for avoiding core material damage. It is not straightforward to conclude whether the power density of 24.3 $\mathrm{W} / \mathrm{\mu m}^{2}$ would be acceptable or not, only relying on the literature values. However, it is one or two orders of magnitude less than the reported values (measured in pulse regimes $[21,22]$ ), and our estimation indicates that the core temperature rise by the pump and signal power would not be detrimental as long as the fiber is efficiently cooled down by good heat-sinking arrangements. Thus, in terms of power density our fiber should be able to sustain $10 \mathrm{~kW}$ of output power.

As a result, in terms of thermal management, Raman nonlinear scattering, and material damage, we predict that our fiber is capable of reaching $10 \mathrm{~kW}$ of output power with state-of-the-art pump diodes. In any case, an experimental demonstration is needed to conclusively address all possible issues at such a power level.

\section{CONCLUSION}

We have demonstrated a highly efficient, multi-kilowatt double-clad $\mathrm{Yb}^{3+}$-doped large-core fiber laser. Pumppower-limited, continuous-wave output power of in excess of $2.1 \mathrm{~kW}$ at $1.1 \mu \mathrm{m}$ was achieved in a single-mode beam $\left(\mathrm{M}^{2}=1.2\right)$. Our result verifies that the current YDFL technology, including fiber fabrication and diode stack pump sources, are fully capable of producing multikilowatt output power with a diffraction-limited beam out of a large-core single-fiber-based YDF laser. We estimate that with state-of-the-art diode pump sources, more than ten times brighter than the diodes we used, our fiber is power-scalable to $10 \mathrm{~kW}$ of output power in terms of thermal management, Raman nonlinear scattering, and material damage.

\section{ACKNOWLEDGEMENT}

This work was supported in part by JTO under contract FA9451-06-D-0014.

\section{REFERENCES}

1. Y. Jeong, J. Sahu, D. Payne, and J. Nilsson, "Ytterbiumdoped large-core fiber laser with $1.36 \mathrm{~kW}$ continuouswave output power," Opt. Exp. 12, 6088-6092 (2004).

2. G. Bonati, H. Voelckel, U. Krause, A. Tünnermann, J. Limpert, A. Liem, T. Schreiber, S. Nolte, and H. Zellmer, "1.53 kW from a single Yb-doped photonic crystal fiber laser," Late Breaking Developments Session 5709-2a, Photonics West 2005.

3. Information available from http://www.ipgphotonics.com.
4. I.-B. Sohn, Y.-C. Noh, Y.-S. Kim, D.-K. Ko, and J. Lee, "Laser ablation of polypropylene films using nanosecond, picosecond, and femtosecond laser," J. Opt. Soc. Korea 12, 38-41 (2008).

5. Y. Izawa, N. Miyanaga, J. Kawanaka, and K. Yamakawa, "High power lasers and their new applications," J. Opt. Soc. Korea 12, 178-185 (2008).

6. S. Yoo, C. Basu, A. J. Boyland, C. L. Sones, J. Nilsson, J. K. Sahu, and D. N. Payne, "Photodarkening in Yb-doped aluminosilicate fibers induced by $488 \mathrm{~nm}$ irradiation," Opt. Lett. 32, 1626-1628 (2007).

7. M. Engholm and L. Norin, "Preventing photodarkening in ytterbium-doped high power fiber lasers; correlation to the UV-transparency of the core glass," Opt. Exp. 16, 1260-1268 (2008).

8. J. Koponen, M. Söderlund, H. J. Hoffman, D. A. V. Kliner, J. P. Koplow, and M. Hotoleanu, "Photodarkening rate in Yb-doped silica fibers," Appl. Opt. 47, 1247-1256 (2008).

9. Y. Jeong, J. Nilsson, J. K. Sahu, D. N. Payne, R. Horley, L. M. B. Hickey, and P. W. Turner, "Power scaling of single-frequency ytterbium-doped fiber master oscillator power amplifier sources up to $500 \mathrm{~W}$," IEEE J. Select. Topics Quantum Electron. 13, 546-551 (2007).

10. P. Dupriez, A. Piper, A. Malinowski, J. K. Sahu, M. Ibsen, B. C. Thomsen, Y. Jeong, L. M. B. Hickey, M. N. Zervas, J. Nilsson, and D. J. Richardson, "High average power high repetition rate picosecond pulsed fiber master oscillator power amplifier source seeded by a gain-switched laser diode at $1060 \mathrm{~nm}$," IEEE Photon. Technol. Lett. 18, 1013-1014 (2006).

11. Y. Jeong, J. K. Sahu, R. B. Williams, D. J. Richardson, K. Furusawa, and J. Nilsson, "Ytterbium-doped largecore fibre laser with $272 \mathrm{~W}$ output power," Electron. Lett. 39, 977-978 (2003).

12. P. Laperle, C. Paré, H. Zheng, A. Croteau, and Y. Taillon, "Yb-doped LMA triple-clad fiber laser," in Proc. Photonics North 2006 (Québec, Canada, Jun. 2006), paper FB-07-3-3.

13. J. M. Fini, "Bend-resistant design of conventional and microstructure fibers with very large mode area," Opt. Exp. 14, 69-81 (2006).

14. J. K. Sahu, Y. Jeong, D. J. Richardson, and J. Nilsson, "A $103 \mathrm{~W}$ erbium/ytterbium co-doped large-core fiber laser," Opt. Comm. 227, 159-163 (2003).

15. Y. Jeong, J. Nilsson, J. K. Sahu, D. B. S. Soh, P. Dupriez, C. A. Codemard, S. Baek, D. N. Payne, R. Horley, J. A. Alvarez-Chavez, and P. W. Turner, "Singlemode plane-polarized ytterbium-doped large-core fiber laser with $633 \mathrm{~W}$ continuous-wave output power," Opt. Lett. 30, 955-957 (2005).

16. Y. Jeong, S. Yoo, C. A. Codemard, J. Nilsson, J. K. Sahu, D. N. Payne, R. Horley, P. W. Turner, L. Hickey, A. Harker, M. Lovelady, and A. Piper, "Erbium:ytterbium codoped large-core fiber laser with 297-W continuouswave output power," IEEE J. Select. Topics Quantum Electron. 13, 573-579 (2007).

17. Information available from http://www.newport.com.

18. D. C. Brown and H. J. Hoffman, "Thermal, stress, and thermo-optic effects in high average power double-clad silica fiber lasers," IEEE J. Quantum Electron. 37, 
207-217 (2001).

19. Y. Wang, C.-Q. Xu, and H. Po, "Thermal effects in kilowatt fiber lasers," IEEE Photon. Technol. Lett. 16, 63-65 (2004).

20. G. P. Agrawal, Nonlinear Fiber Optics, 3rd ed. (Academic Press, New York, USA, 2001).

21. A. A. Said, T. Xia, A. Dogariu, D. J. Hagan, M. J. Soileau, E. W. Van Stryland, and M. Mohebi, "Measurement of the optical damage threshold in fused quartz," Appl. Opt. 34, 3374-3376 (1995).

22. A. Smith, B. Do, and M. Söderlund, "Deterministic nanosecond laser-induced breakdown thresholds in pure and $\mathrm{Yb}^{3+}$ doped fused silica," Proc. SPIE 6453, 645317645328 (2007).
23. W. Koechner, Solid-state Laser Engineering, 5th ed. (Springer, Berlin, Germany, 1999).

24. Y. Jeong, S. Baek, P. Dupriez, J.-N. Maran, J. K. Sahu, J. Nilsson, and B. Lee, "Thermal characteristics of an end-pumped high-power ytterbium-sensitized erbium-doped fiber laser under natural convection," Opt. Exp. 16, 19865-19871 (2008).

25. Y. Jeong, P. Dupriez, J. K. Sahu, J. Nilsson, D. Shen, W. A. Clarkson, and S. D. Jackson, "Power-scaling of a 975-nm diode-pumped ytterbium sensitized thuliumdoped silica fibre laser operating in the $2 \mu \mathrm{m}$ wavelength range," Electron. Lett. 41, 173-174 (2005). 